\title{
High-sensitivity C-reactive protein, lipid profile, malondialdehyde and total antioxidant capacity in psoriasis
}

\author{
S. RESHMA ${ }^{1}$, K. C. VASUDHA ${ }^{1}$ and A. L. SHYAM PRASAD ${ }^{2}$ \\ ${ }^{1}$ Dept. of Biochemistry, M.S.Ramaiah Medical College, Bangalore, India. \\ ${ }^{2}$ Dept. of Dermatology, M.S.Ramaiah Medical College and Hospital, Bangalore, India. \\ *Corresponding author; E-mail: drreshmakiran@yahoo.co.in; Bangalore - 560054, Karnataka, India.
} Tel: 09845480008

\begin{abstract}
Psoriasis is a chronic inflammatory skin disease characterized by epidermal hyperproliferation and lymphocytic infiltration. The ongoing inflammatory process in psoriasis affects the arterial wall promoting the atherosclerotic process, increasing the risk of cardiovascular disease in a younger group of patients. Atherosclerotic processes involve an inflammatory component and high-sensitivity C-reactive protein considered as a marker of the inflammatory component in atherosclerosis. This study was undertaken to evaluate the levels of hsCRP and the traditional risk markers like lipid profile in psoriasis. A case control study was done with 40 cases of psoriasis and 40 healthy controls. The biochemical parameters: lipid profile, hsCRP, malondialdehyde and total antioxidant capacity were estimated on the fasting blood samples. There was a significant increase in lipid profile in cases but these levels were well within the reference ranges for the same. There was a significant rise in hsCRP and malondialdehyde with decreased levels of total antioxidant capacity in cases suggesting a state of inflammation and oxidative stress which co-exists with psoriasis and also plays a role in atherosclerosis.
\end{abstract}

() 2011 International Formulae Group. All rights reserved.

Keywords: Inflammation; atherosclerosis; cardiovascular disease; oxidative stress.

\section{INTRODUCTION}

A state of inflammation is known to play a key role in the pathogenesis of a number of chronic inflammatory systemic diseases (CISDs), which include psoriasis, rheumatoid arthritis, systemic lupus erythematosus and Crohn's disease, and also in the pathogenesis of atherosclerosis. Psoriasis is a complex CISD, with environmental and genetic components, that affects the skin, scalp, nails and occasionally the joints, with periods of exacerbation and remission. It is a common disease, with estimates of prevalence ranging from $<1-5 \%$ (Spah, 2008).

The highly specific cellular and molecular responses in atherosclerotic lesions from initial fatty streak to thrombotic complications describe atherosclerosis as an inflammatory disease (Libby et al., 2002).

As psoriasis and atherosclerosis share common pathogenic features, there is an increased relative risk for developing myocardial infarction. Inflammation plays a pivotal link between psoriasis and atherosclerosis. A study by Prodanovich et al. 
(2009) showed a higher percentage of deaths due to atherosclerotic burden among patients with psoriasis than among patients without psoriasis (19.6\% vs $9.9 \%)$.

High sensitivity C-reactive protein (hsCRP), a systemic inflammatory marker, is considered to be an independent risk marker of cardiovascular disease. Increase in the levels of hsCRP in association with other CISD's (rheumatoid arthritis and systemic lupus erythematosus) have enhanced the cardiovascular risk resulting in shorter life expectancy (Wong et al., 2003).

Malondialdehyde is an indicator of oxidative stress. Damage produced by reactive oxygen species (ROS) may be prevented by antioxidants which can be measured by total antioxidant capacity. Oxidative stress may be a determinant of CRP levels and promote proatherosclerotic inflammatory processes in the early stages of cardiovascular disease development.

HsCRP concentrations predict vascular risk even when cholesterol concentrations are low and also patients with low LDL-C and high hsCRP are at a higher risk of future coronary events (Ridker et al., 2002). Previous studies have attributed CRP as a prognostic disease marker in psoriasis (Rocha-Pereira et al., 2004; Christopher et al., 2010). However studies attributing the elevated levels of hsCRP to cardiovascular risks and events in psoriasis are scarce.

The present study attempts to know the levels of serum hsCRP, malondialdehyde and total antioxidant capacity in patients with psoriasis comparing it with the traditional risk factors for atherosclerosis like the lipid profile.

\section{MATERIALS AND METHODS}

A case-control study was undertaken. 40 clinically diagnosed cases of chronic plaque psoriasis in the active phase of the disease attending the Dermatology Outpatient Clinic and 40 age and sex matched healthy controls who came for a master health checkup at M.S. Ramaiah Medical Teaching Hospital were recruited.

The cases and the controls were in the age group of 15-45 years of both sexes. The cases included were only those on topical therapy. Patients with past or present history of smoking, diabetes mellitus, hypertension, ischemic heart disease, chronic liver and kidney diseases, acute infections autoimmune diseases, corticosteroids, multivitamin therapy were excluded from the study. The duration of illness with remissions and exacerbations varied between 2 months -15 years.

An oral informed consent was taken from the study subjects before the collection of sample. The ethical clearance was obtained from the institutional ethical review board. 5 $\mathrm{ml}$ of blood sample was collected in the yellow vacutainer bulb containing clotting agent. The blood sample were collected after an 8-10 hr of fasting and the separated serum was analyzed on the auto-analyser DADE BEHRING for levels of fasting blood glucose (to exclude diabetic patients) (Henry, 1974) and fasting lipid profile (serum total cholesterol (Stadtman, 1957), serum triglycerides (Burtis, 1994) and HDL cholesterol (Warnick, 1995). LDL cholesterol was calculated by Friedwald's Equation (Friedewald et al., 1972). Serum hsCRP was measured by immunoturbidimetric method by Quantia-CRP US on a semi auto-analyser (Hamwi, 2001).

Serum Malondialdehyde (MDA) was measured by Thiobarbituric acid reactive substances (TBARS) method (Wilbur, 1943), Total antioxidant capacity (TAC) by ferric reducing ability of plasma (FRAP) assay (Benzie, 1996) on ELICO Spectrophotometer. The statistical analysis was done by Student t- test (two tailed, independent). A p 
value $<0.05$ was taken as significant. Receiver Operating Characteristics (ROC) curve analysis was carried out to assess the hsCRP as marker for prediction of atherosclerosis in psoriasis. Pearson correlation between hsCRP and cardiovascular risk variables in cases and controls was done. Chi-square / Fisher Exact test was used to find the significance of study parameters on categorical scale between two or more groups. Effect size was calculated to estimate the effect in study group.

\section{RESULTS}

Results are shown in the Tables 1, 2, 3 and 4. Table 1 shows comparison of lipid profile parameters between controls and cases. Serum total cholesterol, LDL cholesterol, LDL/HDL, total cholesterol/HDL ratios showed a significant increase with a significant decrease in HDL cholesterol in cases but the mean levels of these parameters were well within the reference ranges for the same (NIH Publication, 2002).

Table 2 shows mean levels and standard deviation of hsCRP in cases as $4.74 \pm 1.83$ when compared to $1.78 \pm 0.91$ of controls with a strongly significant 'p'value of $<0.001$. The second parameter in the table shows the mean levels and standard deviation for total antioxidant capacity in cases as $334.46 \pm 97.41$ when compared to controls with 405.99 \pm 127.72 . This had a significant 'p'value of 0.006 . The third parameter in the table, malondialdehyde, showed mean levels and standard deviation of $2.98 \pm 0.85$ in cases when compared to $1.45 \pm 0.83$ in the control group with a significant 'p'value of $<0.001$.

Table 3 and Figure 1 show diagnostic assessment for hsCRP as a marker of atherosclerosis in psoriasis. The area under ROC curve for hsCRP was 0.9541 which indicated that it is an excellent test in psoriasis for cardiovascular risk assessment.

Table 4 shows Pearson correlation of hsCRP with cardiovascular risk profile. In the controls, hsCRP showed significantly strong negative correlation with HDL ( $p<0.001)$, positive correlation with triglycerides $(\mathrm{p}=$ 0.048), LDL/HDL ratio ( $\mathrm{p}=0.024), \mathrm{TC} / \mathrm{HDL}$ $(\mathrm{p}=0.004)$ and with MDA $(\mathrm{p}=0.024)$. In cases, the hsCRP showed negative correlation with HDL and positive correlation with other parameter. This was however not significant.

Table 1: Comparison of lipid profile parameters in cases and controls.

\begin{tabular}{lcccc}
\hline Variables & $\begin{array}{c}\text { Control } \\
(\text { mean } \pm \text { S.D })\end{array}$ & $\begin{array}{c}\text { Cases } \\
(\text { mean } \pm \text { S.D })\end{array}$ & Significance & $\begin{array}{c}\text { Effect } \\
\text { size }\end{array}$ \\
\hline Total cholesterol (mg/dL) & $143.43 \pm 27.5$ & $163.48 \pm 38.65$ & $\mathrm{t}=2.673 ; \mathrm{p}=0.009 * *$ & $2.00(\mathrm{VL})$ \\
HDL $(\mathrm{mg} / \mathrm{dL})$ & $47.45 \pm 9.45$ & $39.60 \pm 6.98$ & $\mathrm{t}=4.226 ; \mathrm{p}<0.001 * *$ & $0.94(\mathrm{~L})$ \\
Triglycerides $(\mathrm{mg} / \mathrm{dL})$ & $107.60 \pm 25.97$ & $135.80 \pm 120.52$ & $\mathrm{t}=1.447 ; \mathrm{p}=0.152$ & $0.32(\mathrm{~S})$ \\
LDL $(\mathrm{mg} / \mathrm{dL})$ & $77.08 \pm 34.45$ & $98.03 \pm 33.26$ & $\mathrm{t}=2.749 ; \mathrm{p}=0.007 * *$ & $0.61(\mathrm{M})$ \\
LDL/HDL & $1.67 \pm 0.69$ & $2.50 \pm 0.85$ & $\mathrm{t}=4.759 ; \mathrm{p}<0.001^{* *}$ & $1.06(\mathrm{~L})$ \\
Total cholesterol/HDL & $3.13 \pm 0.81$ & $4.22 \pm 1.32$ & $\mathrm{t}=4.423 ; \mathrm{p}<0.001^{* *}$ & $0.98(\mathrm{~L})$ \\
\hline VL: Ver
\end{tabular}

VL: Very large; L: Large; M: Medium; S: Small. 
Table 2: hsCRP, MDA and TAC in cases and controls.

\begin{tabular}{lcccc}
\hline Parameters & $\begin{array}{c}\text { Control } \\
(\text { mean } \pm \text { S.D })\end{array}$ & $\begin{array}{c}\text { Cases } \\
(\text { mean } \pm \text { S.D })\end{array}$ & Significance & Effect size \\
\hline hsCRP $(\mathrm{mg} / \mathrm{L})$ & $1.78 \pm 0.91$ & $4.74 \pm 1.83$ & $\mathrm{t}=9.157 ; \mathrm{p}<0.001^{* *}$ & $2.03(\mathrm{VL})$ \\
TAC $(\mu \mathrm{mol} / \mathrm{L})$ & $405.99 \pm 127.72$ & $334.46 \pm 97.41$ & $\mathrm{t}=2.817 ; \mathrm{p}=0.006 * *$ & $0.62(\mathrm{M})$ \\
MDA $(\mu \mathrm{mol} / \mathrm{L})$ & $1.45 \pm 0.83$ & $2.98 \pm 0.85$ & $\mathrm{t}=8.161 ; \mathrm{p}<0.001^{* *}$ & $1.61(\mathrm{VL})$ \\
\hline \multicolumn{2}{l}{ VL: Very large; M: Medium. } & & &
\end{tabular}

Table 3: Diagnostic assessment of hsCRP as a marker of atherosclerosis in psoriasis based on ROC analysis.

\begin{tabular}{lccccccc}
\hline ROC for hSCRP & AUROC & SE & Sensitivity & Specificity & Accuracy & LR+ & LR- \\
\hline$(>=2.2)$ & 0.954 & 0.02 & $100.00 \%$ & $57.50 \%$ & $78.75 \%$ & 2.35 & 0.00 \\
$(>=2.5)$ & 0.954 & 0.02 & $97.50 \%$ & $60.00 \%$ & $78.75 \%$ & 2.44 & 0.04 \\
$(>=2.6)$ & 0.954 & 0.02 & $95.00 \%$ & $72.50 \%$ & $83.75 \%$ & 3.45 & 0.07 \\
$(>=2.7)$ & 0.954 & 0.02 & $90.00 \%$ & $80.00 \%$ & $85.00 \%$ & 4.50 & 0.13 \\
$(>=2.8)$ & 0.954 & 0.02 & $85.00 \%$ & $87.50 \%$ & $86.25 \%$ & 6.80 & 0.17 \\
$(>=2.9)$ & 0.954 & 0.02 & $82.50 \%$ & $90.00 \%$ & $86.25 \%$ & 8.25 & 0.19 \\
$(>=3)$ & 0.954 & 0.02 & $80.00 \%$ & $90.00 \%$ & $85.00 \%$ & 8.00 & 0.22 \\
$(>=3.1)$ & 0.954 & 0.02 & $77.50 \%$ & $95.00 \%$ & $86.25 \%$ & 15.50 & 0.24 \\
\hline
\end{tabular}

§AUROC: Area under ROC; LR: Likelihood ratio.

Table 4: Pearson correlation of hsCRP with other cardiovascular risk variables in cases and controls.

\begin{tabular}{lcccc}
\hline \multirow{2}{*}{ Pair } & \multicolumn{2}{c}{ Control } & \multicolumn{2}{c}{ Cases } \\
\cline { 2 - 5 } & r value & p value & r value & p value \\
\hline hsCRP vs Total cholesterol & 0.146 & 0.368 & 0.080 & 0.171 \\
hsCRP vs HDL & -0.510 & $0.001 * *$ & -0.171 & 0.291 \\
hsCRP vs Triglycerides & 0.314 & $0.048^{*}$ & -0.008 & 0.960 \\
hsCRP vs LDL & 0.298 & 0.062 & 0.142 & 0.387 \\
hsCRP vs LDL/HDL & 0.357 & $0.024^{*}$ & .0 .217 & 0.185 \\
hsCRP vs TC/HDL & 0.442 & $0.004^{*}$ & 0.157 & 0.334 \\
hsCRP vs TAC & 0.012 & 0.941 & -0.171 & 0.292 \\
hsCRP vs MDA & 0.357 & $0.024^{*}$ & 0.127 & 0.434 \\
\hline * Moderately significant $(\mathrm{P}$ value: $0.01<\mathrm{P} \leq 0.05)$ & & & &
\end{tabular}




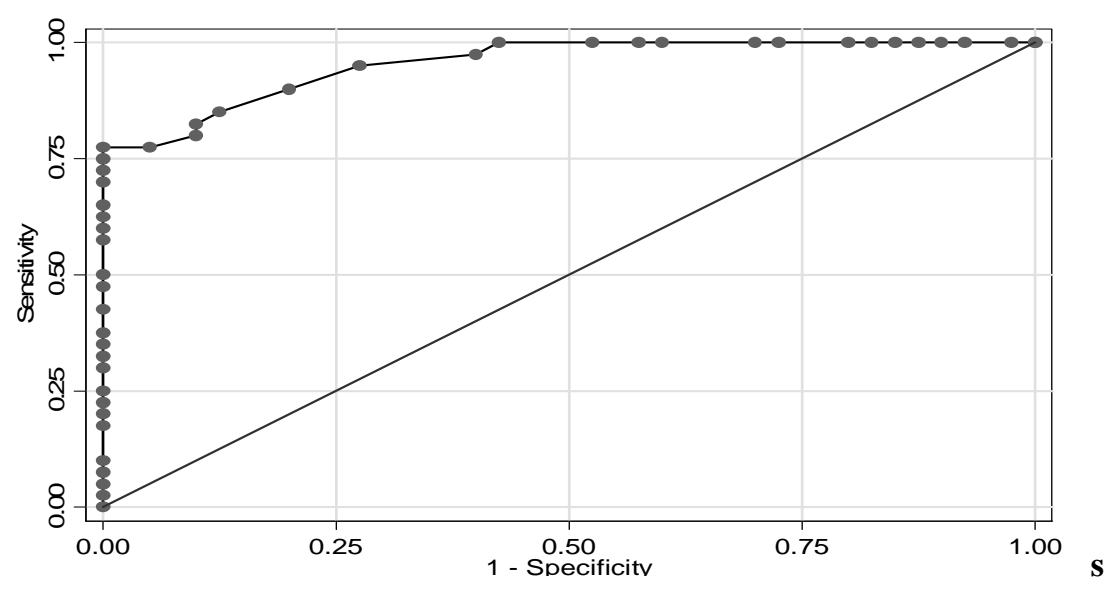

Figure 1: ROC analysis for hsCRP as a diagnostic test for atherosclerosis in psoriasis. Diagnostic values based on Area under curve: 0.9-1.0 Excellent test; 0.8-0.9 Good test; 0.7-0.8 Fair test; 0.6-0.7 Poor test; 0.5-0.6 Fail

$\S$ Area under curve $=0.9541$

\section{DISCUSSION}

Psoriasis is associated with premature atherosclerosis and increased prevalence of coronary artery disease, cerebrovascular and peripheral artery disease due to chronic inflammatory processes (Ludwig, 2007). The increased risk is due to the effects of chronic inflammatory changes, particularly the infiltration of $\mathrm{T}$ cells and the subsequent secretion of pro-inflammatory cytokines. Psoriasis is considered a Th1/Th17 inflammatory disease. It is also seen that Th17 cell response plays an important role in cardiovascular diseases. IL-17 which is produced by activated CD4+ T-cells acts synergistically to elicit further production of pro-inflammatory cytokines by the keratinocytes. IFN $\gamma$ and TNF $\alpha$ induce keratinocytes to produce IL-6, IL-7, IL-8, IL12, IL-15, IL 18 and TNF $\alpha$ in addition to several other cytokines, chemokines and growth factors. IFN $\gamma$ is an important mediator of inflammation in both psoriasis and atherosclerosis and can stimulate the expression of MHC class II molecules and
ICAM-1. Thus, the production of proinflammatory cytokines, together with the activation of inflammatory cells, could contribute to the development of both psoriatic and atherosclerotic lesions (Ramin, 2010). Chronic systemic non-vascular infection is pro-atherogenic and acute systemic inflammatory episodes are markedly associated with atherosclerotic processes.

It is seen that many individuals develop coronary artery disease and other forms of cardiovascular disease in the absence of abnormalities in the lipid profile. Based on the evidence supporting a role of inflammation in the pathogenesis of atherosclerosis, serum markers of inflammation have gained interest as risk markers for atherosclerosis.

Previous studies of lipid profile in psoriasis that have been carried out show an aberrant lipid profile however not consistent. A study by Rocha-Pereira et al. (2004) showed an increase in TC, LDL, and TG with a decrease in HDL cholesterol levels in psoriasis compared to controls. A study by Uyanik et al. (2002) showed no differences in 
TC, HDL, LDL levels with an increase in TG in the study group. Seishima et al. (1994) showed no differences in TC and HDL levels with increased levels of TG in cases compared to controls.

According to the AHA-CDC (American Heart Association-Centre for Disease Control) (2003), hsCRP of $>3 \mathrm{mg} / \mathrm{L}$ is considered as high risk for cardiovascular diseases. In the present study, serum hsCRP levels in most of the patients was $>3 \mathrm{mg} / \mathrm{L}$ and therefore fall under the high risk category.

Skin is a major target of oxidative stress. Although the endogenous antioxidants can oppose the harmful effects of ROS, increased or prolonged presence of free radicals can override ROS defense mechanism and mediate cellular responses that contribute to the development of skin lesions of psoriasis. The lipid oxidation marker (MDA which is measured as TBARS) was strongly associated with cardiovascular events which included unstable angina, fatal/nonfatal myocardial infarction and stroke (Mary et al., 2002).

A study by Rocha-Pereira et al. (2004) showed that the lesions of psoriasis worsened in association with the enhancement of oxidative stress suggesting that patients with psoriasis should be considered as a group at risk for cardiovascular disease.

In this study, LDL-cholesterol was found to be within normal ranges in the cases suggesting that the native-LDL oxidation may become the proatherogenic factor owing to the oxidative stress. There was significant correlations between hsCRP and the other parameters in the control group but though the correlations were obtained in the cases, it was not significant probably due to the underlying disease and it would imply that hsCRP could serve as an independent risk marker and may not be used in conjunction with traditional risk markers in psoriasis.
Similar study has been done in other known chronic inflammatory systemic diseases like rheumatoid arthritis and inflammatory polyarthritis where a prospective follow up showed that baseline elevation of CRP in patient group had a higher incidence of death from cardiovascular events within a period of 10 years (Goodson et al., 2005).

A study showed there was an increase in arterial stiffness (as measured by brachial to ankle pulse wave velocity (baPWV) and digital hyperaemic response measured using the peripheral arterial tonometry (PAT) index) in young psoriatic patients which correlated positively with hsCRP, suggesting the role of systemic inflammation and its association with premature atherosclerosis (Yiu KH et al., 2011).

Contrary to the previous studies which have estimated CRP in CISD, the present study evaluates the usefulness of the estimation of hsCRP in comparison with the traditional risk markers for atherosclerosis in psoriasis. CRP which is secreted in response to IL-6, is a powerful proatherogenic factor in addition to being a risk marker of atherosclerotic events because it contributes to the underlying endothelial dysfunction, as it directly upregulates endothelial cell adhesion molecules, such as ICAM-1, VCAM-1, and Eselectin facilitating the leukocyte-endothelial interaction, which is an early step in atherogenesis (Verma et al., 2002).CRP uncouples eNOS, resulting in increased superoxide production, decreased NO production, and altered eNOS phosphorylation. CRP functions to upregulate the transcription factor $\mathrm{NF}-\kappa \mathrm{B}$ which has been implicated as a key mediator of atherosclerosis (Devaraj et al., 2004). Uptake of oxidized LDL by macrophages is an important process in atherogenesis contributing to plaque progression. CRP 
promotes this uptake due to unexposed phosphocholine epitopes on OxLDL (Zwaka et al., 2002).

As CRP is also known to have direct pro-inflammatory effects on the endothelial cells, lowering serum CRP levels may have beneficial effects on the evolution of atherosclerosis, thus reducing the risk of cardiac events. This could be done by statin therapy (Steven et al., 2005). The JUPITER trial showed that patients with moderate cardiovascular disease risk, with LDL cholesterol levels below $3.4 \mathrm{mmol} / \mathrm{L}$ and hsCRP levels greater than $2 \mathrm{mg} / \mathrm{L}$, with highdose rosuvastatin reduced the number of CVD end points (Ridker PM et al., 2009).

An alternative and highly attractive agent that could be used to decrease inflammatory biomarkers like hsCRP and therefore reduce atherothrombosis would be low-dose methotrexate (LDM). Incidentally, methotrexate, an immunosuppressant, is widely used in psoriasis as a systemic modality of treatment (Solomon, 2006).

The present study has shown that the traditional risk factors can be well within established reference ranges while the hsCRP levels are already elevated.

That inflammation plays a fundamental role in atherothrombosis and that hsCRP is a clinically effective predictor and a proatherogenic factor have well been proved. The attributable risk of coronary artery disease associated with inflammation is as high as that associated with hyperlipidemia and hsCRP. If systemic inflammation promotes atherosclerosis, then the use of anti-inflammatory agents in psoriasis may decrease the cardiovascular disease burden in this population. Contrary to the observations made in the previous studies, this cross-sectional study compares hsCRP with lipid profile (conventional cardiovascular risk markers). This is a pilot study open to research. A follow up study on a larger group of psoriatic patients with/without atherosclerosis (diagnosed by invasive techniques) may be able to prove the role of hsCRP as an inexpensive risk marker of atherosclerosis to predict future cardiovascular events in psoriatic patients.

\section{ACKNOWLEDGEMENTS}

Dr. K. P. Suresh, Scientist (Biostatistics), NIANP (ICAR), Bangalore for the statistical analysis.

\section{REFERENCES}

Benzie FF, Strain JJ. 1996. The ferric reducing ability of plasma (FRAP) as a measure of antioxidant power-The FRAP assay. Analytical Biochemistry, 239: 7076.

Burtis CA, Ashwood ER.1994. Tietz Textbook of Clinical Chemistry (2nd edn). W.B. Saunders Co: Philadelphia; 1017.

Centers for Disease Control and Prevention and the American Heart Association. 2003 Markers of inflammation and cardiovascular disease: application to clinical and public health practice: A statement for healthcare professionals from the Centers for Disease Control and Prevention and the American Heart Association. 107(3): 499-511.

Christopher Ritchlin T, Abrar A, Qureshi, Kurt de Vlam, Costantino Pitzalis, Philip Helliwell. 2010. Biomarkers in Psoriasis and Psoriatic Arthritis: GRAPPA 2008. The Journal of Rheumatology, 37(2): 462467.

Devaraj S, Kumaresan PR, Jialal I. 2004. Effect of C-reactive protein on chemokine expression in human aortic endothelial cells. J Molecular Cell Cardiology, 36: 405-410.

Friedewald WT, Levy RI, Fredrickson DS. 1972. Estimation of the concentration of low-density lipoprotein cholesterol in 
plasma, without use of the preparative ultracentrifuge. Clinical Chemistry, 18(6): 499-502.

Goodson NJ, Deborah Symmons PM, David Scott, Diane Bunn, Mark Lunt, Alan Silman J. 2005. Baseline levels of Creactive protein and prediction of death from cardiovascular disease in patients with inflammatory polyarthritis: a tenyear follow up study of a primary carebased inception cohort. Arthritis Rheum., 52(8): 2293-9.

Hamwi A, Vukovich T. 2001. Evaluation of turbidimetric high sensitivity C-reactive protein assaya for cardiovascular risk estimation. Clinical Chemistry, 47: 20442046.

Henry RJ. 1974. Clinical Chemistry Principles and Techniques (2nd edn). Harper and Row: New York; 1283.

Libby P, Paul M. 2002. Ridker:Inflammation and Atherosclerosis. Circulation, 105: 1135.

Ludwig RJ, Herzog C, Rostock A. 2007. Psoriasis: a possible risk factor for development of coronary artery calcification. Br J Dermatol., 156: 271276.

NIH Publication. 2002. National Cholesterol Education Program. Third report of the National Cholesterol Education Program (NCEP) Expert Panel on Detection, Evaluation and Treatment of high blood cholesterol in adults (ATP III) Final Report.: No.02-5215.

Nissen SE, Tuzcu M, Schoenhagen P, Crowe T, Sasiela WJ, Tsai J. 2005. Statin therapy, LDL cholesterol, C-reactive protein, and Coronary Artery Disease. $N$ Engl J Med., 352: 29-38.

Prodanovich S, Kirsner RS, Kravetz JD, Fangchao, Martinez L, Federman DG. 2009. Association of psoriasis with coronary artery, cerebrovascular and peripheral vascular diseases and mortality. Arch Dermatol. , 145(6): 700-703.

Ramin Ghazizadeh, Hajime Shimizu, Mamiko Tosa, Mohammad Ghazizadeh. 2010. Pathogenic mechanisms shared between psoriasis and cardiovascular disease. Int J Med Sci., 7: 284-289.

Ridker PM, Nader R, Lynda R, Buring JE, Nancy R. 2002. Comparison of C-reactive protein and low-density lipoprotein cholesterol levels in the prediction of first cardiovascular events. $N$ Engl J Med., 347: 1557-65.

Ridker PM, McFayden JG, Fonseca FA, Genest J, Gotto AM, Kastelein JJ, Koenig W, Libby P, Lorenzatti AJ, MacFadyen JG, Nordestgaard BG, Shepherd J, Willerson JT, Glynn RJ. 2009. Number needed to treat among men and women with low low-density lipoprotein cholesterol and elevated high-sensitivity C-reactive protein: Justification for the Use of Statins in Prevention: an Interventional Trial Evaluating Rosuvastatin (JUPITER). Circ Cardiovasc Qual Outcomes, 2(6): 616623.

Rocha-Pereira P, Santos-Silva A, Rebelo I, Figueiredo, Quintanilha A, Teixeira F. 2004. The Inflammatory Response in Mild and in Severe Psoriasis. The British Journal of Dermatology, 150(5): 917-28.

Seishima M, Seishima M, Mori S. 1994. Serum lipid and apolipoprotein levels in patients with psoriasis. British Journal of Dermatology, 130: 738-742.

Spah F. 2008. Inflammation in atherosclerosis and psoriasis: common pathogenic mechanisms and the potential for an integrated treatment approach. British Journal of Dermatology, 159(2): 10-17.

Solomon DH, Avorn J, Katz JN, Weinblatt ME, Setoguchi S, Levin R, Schneeweiss S. 2006. Immunosuppressive medications 
and hospitalization for cardiovascular events in patients with rheumatoid arthritis. Arthritis Rheum., 54: 3790-3798.

Stadtman TC. Methods in Enzymology (vol III), Colowick SP, Caplan NO (eds). 1957. Academy Press: New York; 392394, 678-691.

Uyanik BS, Ari Z, Onur E. 2002. Serum lipids and apolipoproteins in patients with psoriasis. Clin Chem Lab Med., 40: 6568.

Verma S, Li SH, Badiwala MV, Weisel RD. 2002. Endothelin antagonism and interleukin-6 inhibition attenuate the proatherogenic effects of C-reactive protein. Circulation, 105: 1890-1896.

Walter MF, Jacob RF, Jeffers B, Ghadanfar MM, Preston GM, Buch J. 2004. Serum levels of thiobarbituric acid reactive substances predict cardiovascular events in patients with stable coronary artery disease. J Am Coll Cardiol., 44: 19962002.

Warnick GR, Wood PD. 1995. National Cholesterol Education Program recommendations for measurement of high-density lipoprotein cholesterol: Clinical Chemistry, 41: 1427-1433.

Wilbur KM, Bergheim F. 1943. The TBARS reagent as test for the oxidation of unsaturated fatty acids by various agents. Arch. Biochem Biophy., 24: 305-313.

Wong M, Toh L, Wilson A, Rowley K. 2003. Reduced arterial elasticity in rheumatoid arthritis and the relationship to vascular disease risk factors and inflammation. Arthritis Rheum., 48: 81-89.

Yiu KH, Yeung CK, Chan HT, Wong R, Tam S, Lam KF, Yan GH, Yue WS, Chan HH, Tse. 2011. Increased Arterial Stiffness in Patients with Psoriasis is Associated with Active Systemic Inflammation. The British Journal of Dermatology, 14(3): 514-520.

Zwaka TP, Hombach V, Torzewski J. 2000. C-reactive protein-mediated low density lipoprotein uptake by macrophages: implications for atherosclerosis. Circulation, 103: 1194-1197. 